Rev. Geol. Amér. Central, vol. esp. Terremoto de Limón: 139-152

\title{
LOS DESLIZAMIENTOS CAUSADOS POR EL TERREMOTO DE LIMON: FACTORES DE CONTROL Y COMPARACION CON OTROS EVENTOS EN COSTA RICA
}

\author{
Sergio Mora' $\&$ Rolando Mora ${ }^{2}$ \\ 'Dept. Geología, I.C.E., FAX: 506231 4744, Escuela C.A. Geología, Univ.Costa Rica \\ ${ }^{2}$ Escuela Centroamericana de Geología, Apdo. 35, 2060 Universidad de Costa Rica
}

\begin{abstract}
The April 22, 1991 Limón earthquake, as others in recent history in Costa Rica, has triggered extensive landslides. Their most important concentration occured in the viccinity of the epicentral area, on steep slopes covered with residual-regolithic soils originated from Tertiary sedimentary and intrusive rocks. An excentrical distribution of the most important landslide areas in relation to the epicenter is observed. This is probably due to the tectonic setting, rupture propagation, wave directivity and geomorphicconditions of the region. Areas affected with landslides coincide with those with higher values of the Relative Relief Index. Concentration of local faults and amplification of seismic vibrations on high and steep mountains also contributed to increase landslide coverages. Most landslides could be classified as regolithic flows and rock falls although rock-earth slumps and slump-earth flows also occured. Considering that almost $2000 \mathrm{~km} 2$ were affected, most of which orginally covered by tropical rainforest, this event caused a major environmental disaster from which it will take several decades to recover. By using available data, this event has been compared with others in Costa Rica since 1888. Correlations between magnitudes, intensities and epicentral distances show that local reliefs are responsible for the magnification of landslide coverages.
\end{abstract}

RESUMEN: El Terremoto de Limón $(22 / 4 / 91, \mathrm{Ms}=7,5)$ ha producido, como otros en la historia reciente de Costa Rica, extensos y severos deslizamientos. La concentración de los deslizamientos más importantes no siempre se presenta en las inmediaciones del área epicentral, aunque sí en las laderas de más fuerte pendiente, usualmente cubiertas de suelos residuales. En el caso de este terremoto, en la región afectada por los deslizamientos (cerca de $2000 \mathrm{~km}^{2}$, las laderas se encuentran formadas por rocas sedimentarias e intrusivas del Terciario con diversos grados de meteorización. Fue observada una marcada excentricidad entre las áreas más severamente afectadas por los deslizamientos y el epicentro original del sismo. Esto se podría explicar por el contexto geotectónico, el proceso de ruptura múltiple, la propagación de la ruptura y la directividad de ondas sísmicas hacia el noroeste. Las características geomorfológicas de la región jugaron un papel preponderante, pues las áreas más afectadas por los deslizamientos coinciden con aquellas que poseen valores elevados del Indice de Relieve Relativo. La mayoría de los deslizamientos podrían clasificarse como del tipo "flujo regolítico", pues ocasionaron el desprendimiento y movilización de los horizontes del suelo residual y roca alterada. Su espesor no superó casi nunca los $10 \mathrm{~m}$. Además, se desarrollaron desprendimientos de masas rocosas de suelos. Gran parte de las laderas estaban originalmente cubiertas por bosque tropical primario el cual, en alrededor de $50 \mathrm{~km}^{2}$ quedó casi totalmete destruido. Este es tal vez el mayor desastre ecológico de los últimos tiempos en Costa Rica y tomará quizás varias décadas para recuperarse. La comparación de lo sucedido en este evento con otros anteriores en Costa Rica desde 1888 y de los cuales se dispone de información confiable, ha permitido establecer correlaciones que facilitan la comprensión del desarrollo de los deslizamientos disparados por eventos sísmicos. Se han comparado magnitudes, intensidades, distancias epicentrales, valores del índice de relieve relativo y tasas de destrucción de las laderas). 


\section{INTRODUCCION}

El Terremoto de Limón, ocurrido el 22 de abril de 1991 (Ms = 7,5, prof. 16,5 km), disparó una enorme cantidad de deslizamientos, que afectaron cerca de $2000 \mathrm{~km}^{2}$ y destruyeron alrededor de 50 $\mathrm{km}^{2}$ de bosque tropical húmedo primario, su flora y fauna. Aparte de ello, causaron una erosión subsecuente muy intensa. Como consecuencia, se desarrolló un desequilibrio hidrológico que generó inundaciones, avalanchas de lodo y contaminación con sedimentos de las aguas fluviales, lagunares y marinas. Esto causó severos daños a la población, lineas vitales, actividades productivas y al ambiente.

Esta no es la primera vez en que ha ocurrido un impacto semejante. Los deslizamientos causados por sismos han sido una de las fuentes de daños más importantes causados por la actividad sísmica en Costa Rica. Prácticamente todos los grandes eventos sísmicos han generado deslizamientos, con la excepción del terremoto de Cartago de 1910. La literatura y documentación disponible ha permitido esta conclusión primaria (González, 1910; Leandro et al, 1983; Mora, 1985, 1989; Mora $\&$ Morales, 1986). La figura 1 muestra la secuencia de los eventos sísmicos que han generado importantes deslizamientos desde 1888 .

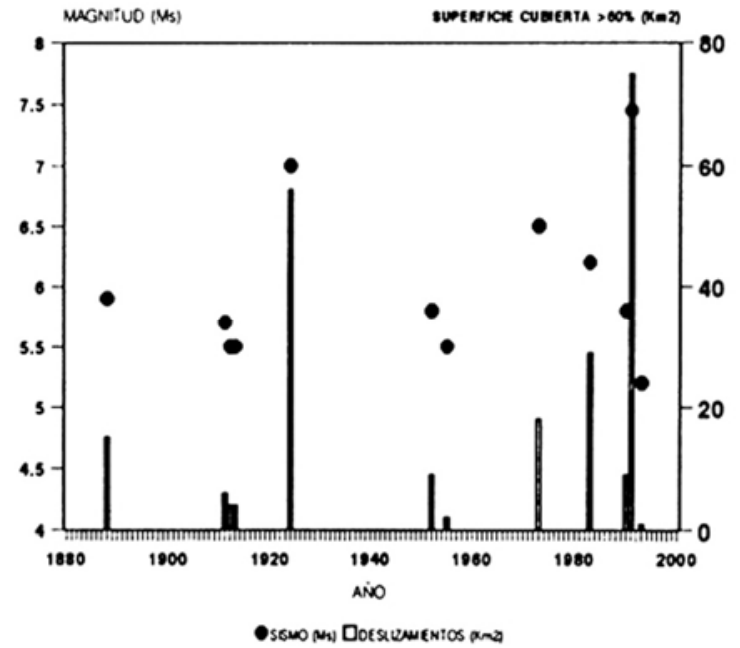

Fig. 1: Secuencia de terremotos que han generado deslizamientos en Costa Rica desde 1888.

Es importante mencionar que los deslizamientos se han presentado con sismos de magnitudes superiores a Ms $=5,2$. Los deslizamientos se presentan, en magnitud y extensión, de acuerdo con las condiciones climáticas (en algunas regiones impactadas por varios eventos sísmicos ha habido mayor cantidad de deslizamientos cuando los sismos han ocurrido durante la estación lluviosa) y geomorfológicas de cada localidad.

Tabla 1

Parámetros de los sismos más importantes que han generado deslizamientos en Costa Rica desde 1888.

\begin{tabular}{|c|c|c|c|c|c|c|c|c|c|}
\hline \multirow[t]{2}{*}{$\begin{array}{l}\text { Fecha } \\
\text { dd/mm/aa }\end{array}$} & \multirow[t]{2}{*}{$\begin{array}{l}\text { Region } \\
\text { epicentral }\end{array}$} & \multirow[t]{2}{*}{$\begin{array}{l}\text { Magnitud } \\
\text { (Ms) }\end{array}$} & \multirow{2}{*}{$\begin{array}{c}\text { Intensidad } \\
\text { (MMmax) } \\
>60 \%\end{array}$} & \multirow{2}{*}{\multicolumn{2}{|c|}{ 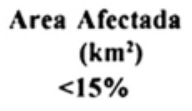 }} & \multicolumn{2}{|c|}{$\begin{array}{c}\text { Dist.Epic.Max. } \\
(\mathrm{km})\end{array}$} & \multicolumn{2}{|c|}{$\underset{\mathbf{m} / \mathbf{k m}^{2}}{\text { Rel.Relat.Min. }}$} \\
\hline & & & & & & $>60 \%$ & $<15 \%$ & $>60 \%$ & $<15 \%$ \\
\hline $30 / 12 / 1888$ & Fraijanes & 5,9 & VIII-IX & 15 & 210 & 7 & 21 & 450 & 350 \\
\hline $10 / 10 / 1911$ & Guatuso & 5,7 & VIII+ & 6 & 90 & 4 & 12 & 475 & 375 \\
\hline 29/08/1911 & Bajos del Toro & 5,5 & VII+ & 4 & 15 & 3 & 15 & 485 & 350 \\
\hline $06 / 06 / 1912$ & Sarchi & 5,5 & VII & 4 & 70 & 3 & 9 & 515 & 375 \\
\hline $04 / 03 / 1924$ & Orotina & 7,0 & IX-X & 56 & 900 & 21 & 50 & 425 & 275 \\
\hline $30 / 12 / 1952$ & Patillos & 5,8 & VIII+ & 9 & 130 & 6 & 18 & 450 & 375 \\
\hline $01 / 09 / 1955$ & Bajos del Toro & 5,5 & VII+ & 2 & 50 & 2 & 8 & 500 & 400 \\
\hline $14 / 04 / 1973$ & Tilarán & 6,5 & IX & 18 & 750 & 19 & 40 & 475 & 225 \\
\hline 03/07/1983 & División-Buvis & 6,2 & VIII-IX & 29 & 550 & 11 & 35 & 500 & 225 \\
\hline May-Dec/90 & Enjamb.Puriscal & Máx=5,8 & VIII & 9 & 390 & 5 & 25 & 500 & 300 \\
\hline $22 / 04 / 1991$ & Limón & 7.45 & VIII-IX & 75 & 1525 & 40 & 80 & 500 & 300 \\
\hline Jul/93 & Enjamb.Pejibaye & Máx=5,2 & VI+ & & 20 & & 10 & & 400 \\
\hline
\end{tabular}




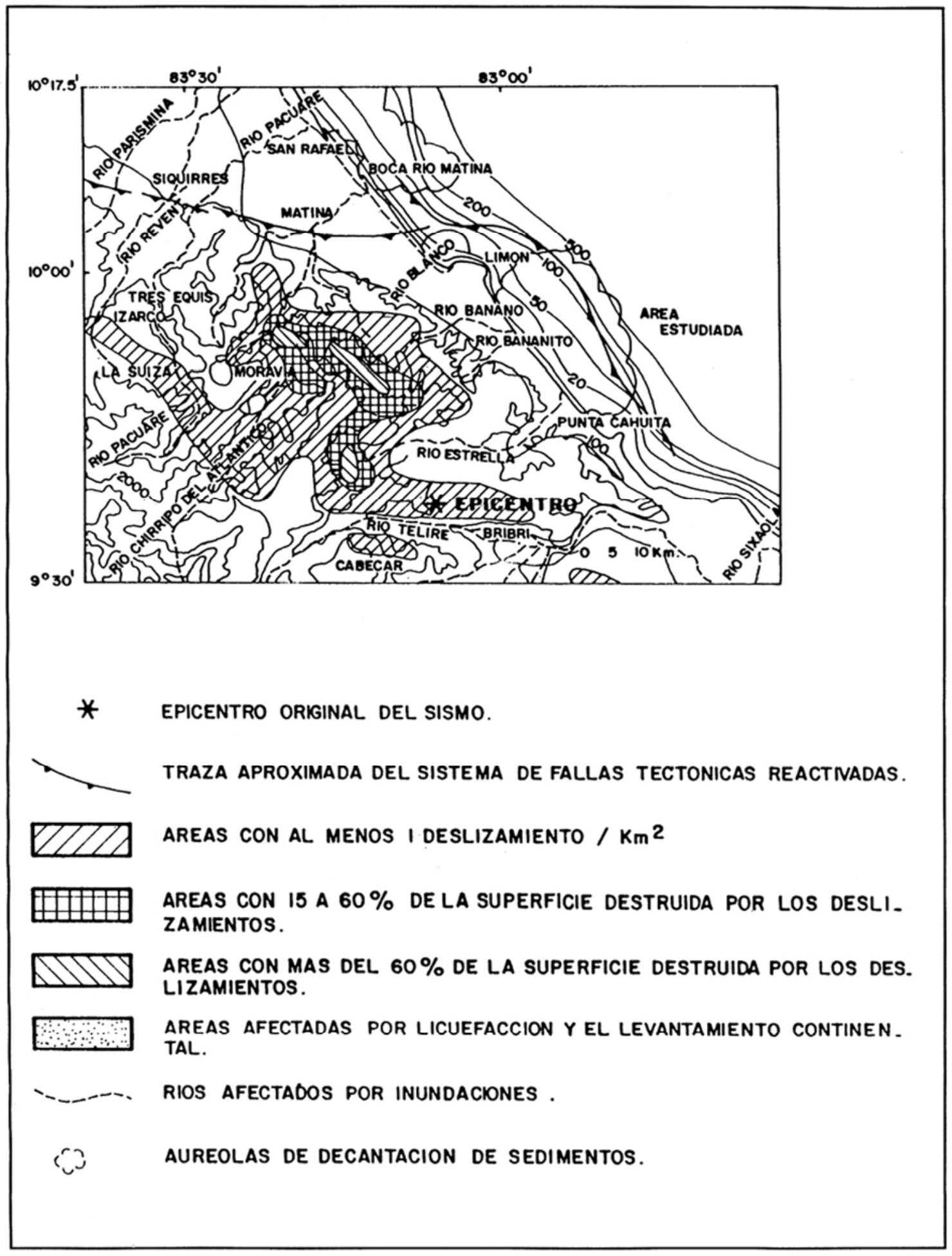

Fig. 2: Mapa de efectos geológicos - geodinámicos generados por el Terremoto de Limón. 
La tabla 1 muestra la lista de eventos sísmicos más importantes que han generado deslizamientos. Se incluyen parámetros, como la magnitud, intensidades máximas en la localidad afectada, las tasas de destrucción (fuente de desprendimiento de materiales, las vías de flujo y las áreas de depositación de sus detritos) causada por los deslizamientos (proporción entre la superficie dañada y la superficie total: $>60 \%, 15-60 \%$ y $<15 \%$, esta última en donde se ha desarrollado al menos un deslizamiento por $\mathrm{km}^{2}$ ), la distancia epicentral y sus índices predominantes de Relieve Relativo (mayor diferencia de elevación por $\mathrm{km}^{2}$, $\mathrm{Rr}=\mathrm{dH} / \mathrm{km}^{2}$, con unidades $\mathrm{m} / \mathrm{km}^{2}$ ).

De la observación de los datos de la tabla 1 es evidente que la rugosidad topográfica del terreno, es decir la pendiente de las laderas, juega uno de los papeles màs importantes en la generación de los deslizamientos. Es por esta razón que se han realizado varias correlaciones en donde se involucra el Indice de Relieve Relativo ya mencionado.

\section{CARACTERISTICAS GENERALES DE LOS DESLIZAMIENTOS DISPARADOS POR EL EVENTO DE 1991}

El área afectada fue dividida en subcuencas y sectores, dentro de los cuales el terreno posee condiciones geomorfológicas, distancias epicentrales, caracteristicas tectónicas, litologías, intensidades sísmicas y usos de la tierra relativamente semejantes (tabla 2).

En términos generales, se ha determinado que en cerca de $2000 \mathrm{~km}^{2}$ ocurrió al menos un deslizamiento por $\mathrm{km}^{2}$ (Fig. 2). Dentro de esta extensión, alrededor de $1525 \mathrm{~km}^{2}$ presentaron al menos un deslizamiento por $\mathrm{km}^{2}$ o hasta una tasa de destrucción en sus laderas de $15 \%$. En cerca de $375 \mathrm{~km}^{2}$ la tasa de destrucción de las laderas alcanzó una proporción de 15 a $60 \%$ de la superficie y en $75 \mathrm{~km}^{2}$ las laderas fueron destruidas en más de un $60 \%$. En alrededor de $25 \mathrm{~km}^{2}$ ocurrió una destrucción prácticamente total, es decir, con una remoción de cerca del $100 \%$ del suelo y la vegetación. Este fue el caso de parte del cañón fluvial del río Chirripó en los alrededores de su confluencia con los ríos Moravia y Boyei y también en algunos sectores de las cuencas altas de los ríos Banano y Bananito.

La mayoría de los deslizamientos se pueden clasificar en las siguientes cuatro categorias definidas por Varnes (1976, en Schuster \& Krizek, 1978), cuya nomenclatura ha sido adaptada a las condiciones locales (Fig.3):

- Deslizamientos superficiales del horizonte regolítico ("regolithic flow"): $69 \%$

Tabla 2

Distribución, por subcuencas y sectores, de las superficies cubiertas por los deslizamientos causados por el sismo de Limón del 22 de abril de 1991.

\begin{tabular}{|c|c|c|c|c|c|c|c|c|c|}
\hline \multirow{2}{*}{$\begin{array}{c}\text { Sector } \\
0 \\
\text { subcuenca }\end{array}$} & \multirow{2}{*}{\multicolumn{3}{|c|}{$\begin{array}{c}\text { Superficie afectada por } \\
\text { deslizamientos }\left(\mathrm{km}^{2}\right) \\
>60 \% \quad 15-60 \%<15 \%\end{array}$}} & \multicolumn{3}{|c|}{$\begin{array}{l}\text { Indice Relieve Relativo } \\
\left(\mathbf{m} / \mathbf{k m}^{2}\right)\end{array}$} & \multicolumn{3}{|c|}{$\begin{array}{l}\text { Dit. Epic. Promedio } \\
\left(\mathbf{k m}^{2}\right)\end{array}$} \\
\hline & & & & $>60 \%$ & $15-60 \%$ & $<15 \%$ & $>60 \%$ & $15-60 \%$ & $<15 \%$ \\
\hline ESTRELLA & 15 & 70 & 180 & $400-600$ & $300-500$ & $200-400$ & 17 & 15 & 15 \\
\hline CABECAR & & & 30 & & & $175-200$ & & & 13 \\
\hline BRIBRI & & & 80 & & & $175-200$ & & & 15 \\
\hline BANANITO & & & 95 & & & $300-350$ & & & 20 \\
\hline RIO BLANCO & & & 20 & & & $350-400$ & & & 33 \\
\hline $\begin{array}{l}\text { BANANO } \\
30\end{array}$ & 40 & 125 & 260 & $450-600$ & $350-500$ & $250-400$ & & 28 & 28 \\
\hline $\begin{array}{l}\text { AYIL-BOYEI } \\
44\end{array}$ & 20 & 80 & 240 & $600-970$ & $450-700$ & $390-500$ & & 40 & 35 \\
\hline CHIRRIPO & & 25 & 305 & & $400-600$ & $275-500$ & & 35 & 34 \\
\hline PACUARE & & & 175 & & & $375-500$ & & & 43 \\
\hline LA SUIZA & & & 90 & & & $400-600$ & & & 55 \\
\hline IZARCO & & & 50 & & & $425-475$ & & & 70 \\
\hline
\end{tabular}




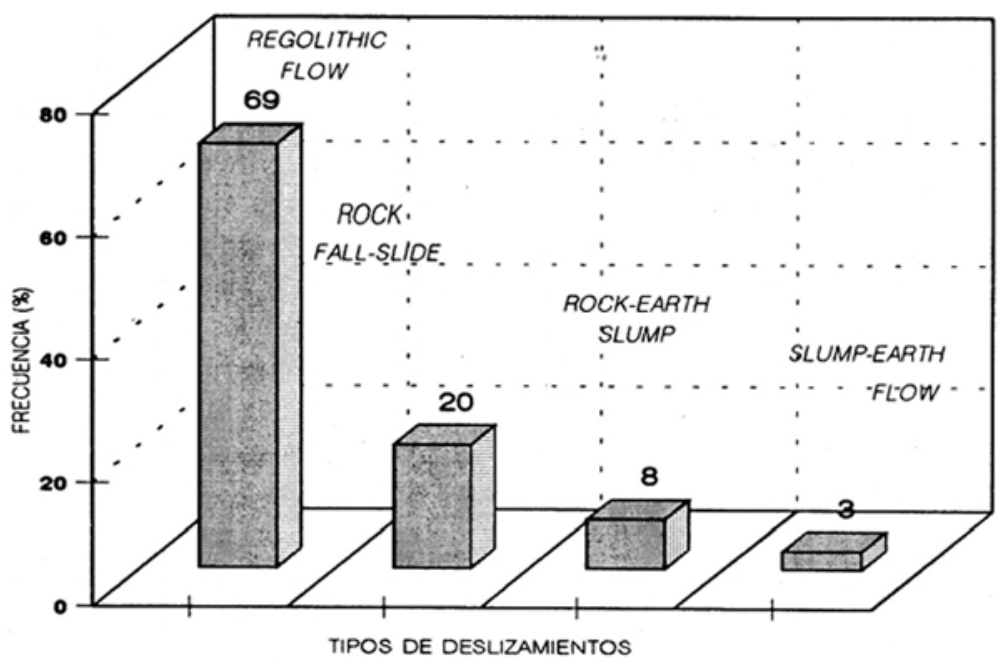

Fig. 3: Distribución de los tipos de deslizamientos más frecuentes generados por el Terremoto de Limón.

- Desprendimiento y deslizamiento de masas rocosas ("rock-fall slide"): $20 \%$

- Deslizamientos traslacionales de suelos y rocas entremezclados ("rock-earth slumps"): $8 \%$

- Deslizamientos traslacionales de masas compuestas predominantemente por suelos ("slump-earthflows"): $3 \%$

Puede agregarse que con el arribo de las lluvias intensas y particularmente con la tormenta tropical de agosto de 1991, se generaron grandes cantidades de avalanchas de lodo y rocas. En muchos casos, la cobertura de deslizamientos aumentó considerablemente a causa de ello y además, los materiales que luego del sismo se mantenían en estabilidad precaria finalizaron por deslizarse a su vez. En esta ocasión, también se formaron numerosos represamientos en los cauces fluviales, tanto de las quebradas pequeñas como de los ríos mayores (Chirripó, Banano y Bananito). La ruptura de estas presas naturales provocó avenidas súbitas ("flash floods") y daños adicionales a la infraestructura, puentes, plantaciones y a la población.

En otras áreas un poco más alejadas (ríos Reventazón y Guayabo, Tres Equis, La Suiza, La Garita de Alajuela, etc.), se reactivaron deslizamientos pre-existentes. Cuando han podido identificarse y discriminarse estos casos de reactivación, no fueron considerados dentro de este estudio. Los deslizamientos ocurridos en el sector panameño de la Cordillera de Talamanca, aparentemente numerosos según las fuentes periodísticas y algunas referencias verbales, tampoco han sido considerados a causa de la ausencia de datos cuantitativos.

\section{CONDICIONES GEOMORFOLOGICAS Y LITOLOGICAS DE LA REGION AFECTADA}

Es realmente escasa la literatura e información general disponibles sobre las litologías, geomorfología y tectónica de la región analizada. Existen tan solo algunos documentos y mapas generalizados y confiables (Escalante, 1983; Chacón, 1994). La región afectada por el sismo y los deslizamientos se encuentra dentro de la vertiente Caribe de Costa Rica. Se trata particularmente del flanco oriental y nororiental de la Cordillera de Talamanca, compuesta por rocas sedimentarias, volcánicas e intrusivas del Terciario y que presentan diferentes grados de meteorización.

\section{Cuenca alta del río La Estrella}

Esta localidad, situada entre 10 y $20 \mathrm{~km}^{2}$ al NNW del epicentro original, está cubierta sobre todo 
de colinas y montañas de mediana altura formadas por rocas sedimentarias plegadas y meteorizadas del Oligoceno y Mioceno. Existen varios sistemas de fallamiento predominantes con direcciones NWSE que controlan la morfología del frente montañoso y de la dirección de los cauces fluviales. Sus suelos residuales (regolíticos) son de considerable espesor, lo que permitió el desarrollo de grandes deslizamientos. Cerca del $20 \%$ de los deslizamientos masivos de tipo traslacional ocurrieron en este sector. También se desarrollaron varias represas efimeras naturales en los cauces, las cuales al romperse, ocasionaron importantes crecidas en el río La Estrella.

En este sector, aproximadamente $15 \mathrm{~km}^{2}$ fueron afectados con deslizamientos que cubrieron más del $60 \%$ de la superficie, especialmente en áreas con relieves relativos superiores a $400 \mathrm{~m} / \mathrm{km}^{2}$. Cerca de $70 \mathrm{~km}^{2}$ tuvieron coberturas de entre $15 \mathrm{y}$ $60 \%$ de deslizamientos y en alrededor de $180 \mathrm{~km}^{2}$ se presentó al menos un deslizamiento por $\mathrm{km}^{2}$, en áreas con relieves de $200 \mathrm{a} 400 \mathrm{~m} / \mathrm{km}^{2}$.

\section{Sector Cabécar}

En este sector, localizado entre 10 y $15 \mathrm{~km}$ al SW del epicentro original, cerca de $30 \mathrm{~km}^{2}$ muestran al menos un deslizamiento por $\mathrm{km}^{2}$, en donde el relieve relativo alcanza valores de 175 a $250 \mathrm{~m} / \mathrm{km}^{2}$. No existen áreas de relieves más fuertes. Las laderas se componen escencialmente de rocas sedimentarias del Terciario plegadas, falladas y con grados avanzados de meteorización. Las fallas locales se presentan con orientaciones predominantes $\mathrm{NE} / \mathrm{SW}$.

\section{Sector Bribri}

La distancia de este sector al epicentro original es de 10 a $35 \mathrm{~km}$. Cerca de $80 \mathrm{~km}^{2}$ mostraron al menos un deslizamiento por $\mathrm{km}^{2}$ en laderas cuyo valor del relieve relativo varía entre 175 y $225 \mathrm{~m} /$ $\mathrm{km}^{2}$. No existen, prácticamente, laderas con valores superiores a estos. Las colinas de la localidad están formadas por rocas sedimentarias del Terciario, plegadas y falladas y con elevados grados de alteración meteórica.

\section{Cuenca superior del río Banano}

Predominan, en este sector, las rocas sedimentarias (areniscas, lutitas) del Terciario con un grado mediano de meteorización. Sin embargo, también se presentan intrusiones de gabro que han causado cierta alteración hidrotermal localizada. De acuerdo con los mapas disponibles, existen aquí también algunas fallas inversas.

Esta parte de la cuenca del río Banano, que fue una delas más severamente afectada por los deslizamientos y se encuentra entre 25 y $35 \mathrm{~km}$ al noroeste del epicentro original. Alrededor de $40 \mathrm{~km}^{2}$ fueron afectados por deslizamientos que cubrieron más del $60 \%$ de la superficie, sobre todo en aquellas áreas en donde los valores del relieve relativo alcanzaron y superaron $450 \mathrm{~m} / \mathrm{km}^{2}$. Otros $125 \mathrm{~km}^{2}$ fueron afectados por deslizamientos que cubrieron entre 15 y $60 \%$ de la superficie, en áreas en donde el relieve alcanzó valores de 350 a $500 \mathrm{~m} / \mathrm{km}^{2}$. En alrededor de $260 \mathrm{~km}^{2}$ se observó al menos un deslizamiento por $\mathrm{km}^{2}$, sobre todo cuando el relieve relativo fue superior a $250 \mathrm{~m} / \mathrm{km}^{2}$.

Los deslizamientos traslacionales masivos fueron menos comunes, pero los flujos regolíticos, desprendimientos y deslizamientos de masas rocosas se presentaron extensamente, en especial en las laderas más empinadas. También fueron observados varios represamientos de cauces fluviales, aunque de pequeñas dimensiones.

\section{Cuencas altas de los ríos Bananito y Blanco}

Las laderas de este sector, localizado entre 20 y $35 \mathrm{~km}$ del epicentro original, se componen de rocas sedimentarias meteorizadas del Terciario superior, intruidas por diques gabroides que han generado pequeñas aureolas de alteración hidrotermal. De la porción del río Bananito, $95 \mathrm{~km}^{2}$ fueron afectados por al menos un deslizamiento por $\mathrm{km}^{2}$ (flujos regolíticos y pequeños deslizamientos traslacionales). Esto ocurrió cuando los valores del relieve relativo alcanzaron entre $300 \mathrm{y}$ $400 \mathrm{~m} / \mathrm{km}^{2}$, los mayores en el sector. Por su parte, en la porción del río Blanco, con condiciones geológicas y de relieve semejantes, fueron afectados cerca de $20 \mathrm{~km}^{2}$ por al menos un deslizamiento por $\mathrm{km}^{2}$. 


\section{Sector Ayil-Boyei}

Este es tal vez el sector en el cual los deslizamientos alcanzaron su desarrollo más espectacular. El relieve del sector es sumamente rugoso, pues alcanza valores de hasta $970 \mathrm{~m} / \mathrm{km}^{2}$, los más elevados de toda la región y entre los más altos de Costa Rica. El efecto de amplificación topográfica de las vibraciones sísmicas es evidente. Además, el espesor de los suelos regolíticos es considerable. Por otra parte, los mapas disponibles muestran una elevada concentración de sistemas de fallamiento tectónico, además de encontrarse en el extremo norte del bloque por el que se propagó la ruptura durante el sismo (directividad y tal vez concentración de la energía de las ondas sísmicas).

Dentro de este sector, cerca de $20 \mathrm{~km}^{2}$, con relieves relativos de 600 a $970 \mathrm{~m} / \mathrm{km}^{2}$, fueron afectados por deslizamientos que cubrieron más del $60 \%$ de la supeficie de las laderas y entre los cuales, en cerca de $15 \mathrm{~km}^{2}$ la destrucción del suelo y la vegetación fue de prácticamente del $100 \%$. La presencia del bosque tropical húmedo, primario y muy denso no fue suficiente para mitigar siquiera el desastre. Los deslizamientos movilizaron los suelos y rocas por debajo del sistema de raíces de los árboles. Cerca de $80 \mathrm{~km}^{2}$, en donde el relieve es de 450 a $700 \mathrm{~m} / \mathrm{km}^{2}$, fue destruido entre $15 \mathrm{y}$ $60 \%$ de su superficie y además se desarrolló al menos un deslizamiento por $\mathrm{km}^{2}$ en alrededor de $240 \mathrm{~km}^{2}$.

La mayoría de los deslizamientos ocurridos en este sector pueden clasificarse como flujos regolíticos y desprendimientos y deslizamientos de masas rocosas. Fueron muy numerosas las avalanchas de lodo y rocas (Capilla 2, Boyei) en las que sus conoides de deyección formaron represas efimeras sobre los cauces fluviales. $\mathrm{Al}$ romperse esas represas, se desarrollaron avenidas súbitas que ocasionaron inundaciones y la migración del cauce del río en varias localidades cercanas a su salida a la llanura.

Un caso interesante de mencionar es el de las Lagunas de Ayil, formadas por el represamiento del río Moravia a causa de un deslizamiento de cerca de 3 millones de metros cúbicos, cerca de su confluencia con el río Chirripó. De acuerdo con la interpretación de las fotografias aéreas de 1945 y
1956 y de algunas referencias (Rudin, M, 1991; comunicación oral), el deslizamiento debe haber ocurrido durante las llevvias intensas asociadas con el huracán Kathy, en octubre de 1954.

Durante este sismo y las lluvias subsiguientes, parte de la presa (alrededor del $25 \%$ ) colapsó y fue removida por los ríos. El resto de la masa, aunque aparenta encontrarse en relativa estabilidad, muestra indicios de una posible desestabilización a mediano o largo plazo (e.g. manantiales posiblemente relacionados con una tubificación y erosión interna de los materiales de la represa, pendientes muy empinadas de los taludes de aguas abajo de la presa, etc.). La laguna más pequeña se ha sedimentado totalmente, mientras que la más grande mantiene tan solo alrededor del $40 \%$ de su volumen original, también a causa de la sedimentación aportada por los deslizamientos en la cuenca superior del río y en las inmediaciones de este sitio.

\section{Cuenca media del río Chirripó}

Se ubica aguas arriba del sector antes mencionado. Aquí, cerca de $25 \mathrm{~km}^{2}$ han sido cubiertos entre 15 y $60 \%$ de la superficie por los deslizamientos, cuando el relieve relativo ha alcanzado valores de $400 \mathrm{a} 600 \mathrm{~m} / \mathrm{km}^{2}$. En relieves de 275 a $500 \mathrm{~m} / \mathrm{K}^{2}$ se ha desarrollado al menos un deslizamiento por $\mathrm{km}^{2}$. Este sector, localizado entre 30 y $40 \mathrm{~km}$ de epicentro original, se compone de rocas sedimentarias clásticas (areniscas, lutitas), rocas volcánicas (tobas, aglomerados, lavas) y algunas intrusiones pequeñas, todas de edad terciaria.

\section{Cuenca media del río Pacuare}

En el área aparecen aglomerados y brechas volcánicas y algunas rocas sedimentarias del Terciario. Se localiza entre 40 y $50 \mathrm{~km}^{2}$ al noroeste del epicentro. Los deslizaminetos, al menos uno por $\mathrm{km}^{2}$, aparecieron en alrededor de $175 \mathrm{~km}^{2}$ cuando los relieves relativos superaron $375 \mathrm{~m} / \mathrm{km}^{2}$ (máximos de $500 \mathrm{~m} / \mathrm{km}^{2}$ ). Casi todos los deslizamientos fueron flujos regolíticos y de dimensiones relativamente moderadas. En este sector se nota un cambio sustancial en el uso de la 
tierra, pues aparecen plantaciones de café, caña de azúcar, pastos, maiz y otros.

\section{Sector La Suiza-Izarco}

Es en esta área que fueron detectados los deslizamientos más alejados del epicentro original (50 a $80 \mathrm{~km}^{2}$ al noroeste de ese puento). Las áreas con al menos un deslizamiento por $\mathrm{km}^{2}$ cubrieron cerca de $90 \mathrm{~km}^{2}$ en los alrededores de La Suiza, mientras que en Izarco alcanzaron alrededor de $\mathbf{5 0}$ $\mathrm{km}^{2}$. Los relieves relativos en este sector varian entre 400 y $600 \mathrm{~m} / \mathrm{km}^{2}$.

Las litologías predominantes son los aglomerados, las rocas sedimentarias y materiales coluvio-aluviales (Mora \& Salazar, 1991). Este sector está prácticamente desprovisto de bosque primario y en su lugar existen extensas plantaciones de café, caña de azúcar, pastos y otros cultivos. Adicionalmente, existe una importante red de caminos y carreteras, en cuyos cortes y rellenos se desarrolaron numerosos deslizamientos

\section{LA DISTRIBUCION DE LOS DESLIZAMIENTOS EN FUNCION DE LA RUGOSIDAD DEL RELIEVE}

Como hasta el momento ha sido mencionado, los deslizamientos ocurrieron en áreas de relieve rugoso, lo cual explica al menos en parte, la excentricidad de las áreas en donde se desarrolló la mayor cantidad de deslizamientos en relación con el epicentro original del sismo. La región epicentral posee en todo caso, un relieve relativamente suave y tan solo ocurrieron unos pocos deslizamientos. Por el contrario, algunas áreas con relieve fuerte, a distancias de $45 \mathrm{~km}$, fueron prácticamente destruidas por los deslizamientos, perdiéndose casi en su totalidad los suelos y la cobertura vegetal.

La forma con la que ocurrió la propagación de la ruptura en el plano de falla, el efecto de directividad de ondas, la composición litológica de las laderas, las condiciones de fallamiento tectónico, meteorización y alteración hidrotermal de cada localidad, la distribución de las réplicas sísmicas y el uso de la tierra podrían haber influenciado la distribución de los deslizamientos y explicar otras singularidades.
La tabla 2 y la figura 4 muestran la tendencia general con la que ocurrieron los deslizamientos en las diversas subcuencas y sectores, al relacionarse la cobertura superficial de los deslizamientos con las distancias epicentrales y los valores del relieve relativo. Puede observarse que las coberturas mayores del $60 \%$ ocurrieron hasta $10 \mathrm{~km}$ de distancia epicentral cuando el relieve alcanzó valores de $>350 \mathrm{~m} / \mathrm{km}^{2}$, o a $40 \mathrm{~km}$ de distancia epicentral si los relieves superan $600 \mathrm{~m} /$ $\mathrm{km}^{2}$. De la misma manera, la aparición de al menos un deslizamiento por $\mathrm{km}^{2}$ se produjo solo para los relieves $>175 \mathrm{~m} / \mathrm{km}^{2}$ a $10 \mathrm{~km}$ de distancia epicentral, mientras que a $70 \mathrm{~km}$ si los relieves superan $\operatorname{los} 500 \mathrm{~m} / \mathrm{km}^{2}$.

\section{COMPARACIONES CON OTROS EVENTOS SISMICOS GENERADORES DE DESLIZAMIENTOS EN COSTA RICA}

Como se ha mencionado, otros eventos sísmicos han generado numerosos y extensos deslizamientos durante la historia reciente de Costa

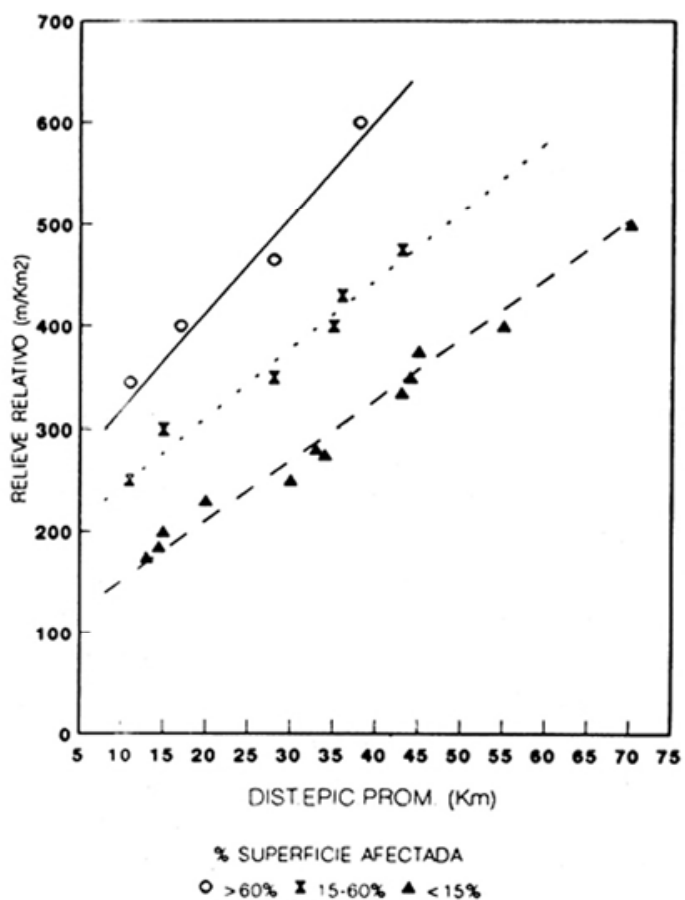

Fig. 4: Relación entre el Indice de Relieve Relativo (Rr) y la distancia epicentral promedio, para cada cobertura de áreas destruidas por deslizamientos. 
Rica, al menos desde 1888 (Fig.1 y tabla 1). En cuanto a las características geomorfológicas y geológicas de las áreas afectadas por estos eventos históricos, se ha podido observar que los deslizamientos aparecen cuando los sismos ocurren con magnitudes (Ms) e intensidades (MM) relativamente moderadas (5.2 y VI respectivamente). Algunos sismos con magnitudes importantes, generalmente superiores a Ms $=6$ como los de $1919,1938,1941,1983$ y 1990 no han generado deslizamientos de relevancia, quizás por sus largas distancias epicentrales hasta las áreas montañosas y por haber ocurrido durante la estación seca.

Es importante recalcar que siete de los doce eventos estudiados tienen sus epicentros dentro o cerca del Valle Central. La vulnerabilidad de esta región, en términos de su elevada población, densidad infraestructural, líneas vitales, actividades productivas y valor económico, le proporciona un nivel de riesgo que debería ser considerado con mayor atención de la que hasta ahora le han prestado las autoridades políticas, los plani. ficadores y los tomadores de decisiones en general. Para comprender mejor sus variables y factores de control se han intentado varias correlaciones, las cuales se describen a continuación.

\section{La intensidad y el relieve relativo}

Al comparar los relieves relativos mínimos, como se aprecia en la figura 5 , es claro que entre más rugoso es el terreno, más fácilmente se desarrollan los deslizamientos. En este caso, para intensidades de VII los deslizamientos aparecen (uno por $\mathrm{km}^{2}$ ) cuando el relieve supera valores de $325 \mathrm{~m} / \mathrm{km}^{2}$. Igualmente, los deslizamientos pueden alcanzar coberturas de destrucción de más del $60 \%$ del área cuando los relieves superan valores de $\mathbf{4 7 5}$ $\mathrm{m} / \mathrm{km}^{2}$.

En caso de que las intensidades sean mayores, por ejemplo de $\mathrm{X}$, se ha podido observar la presencia de al menos un deslizamiento por $\mathrm{km}^{2}$ cuando los relieves superan $\operatorname{los} 200 \mathrm{~m} / \mathrm{km}^{2}$, mientras que las tasas de destrucción mayores de $60 \%$ de la superficie se alcanzan cuando el relieve supera $375 \mathrm{~m} / \mathrm{km}^{2}$. La dispersión apreciable podría explicarse por las diferencias gemorfológicas, litológicas, climáticas y por la calidad de los datos sismológicos de los sismos más antiguos.

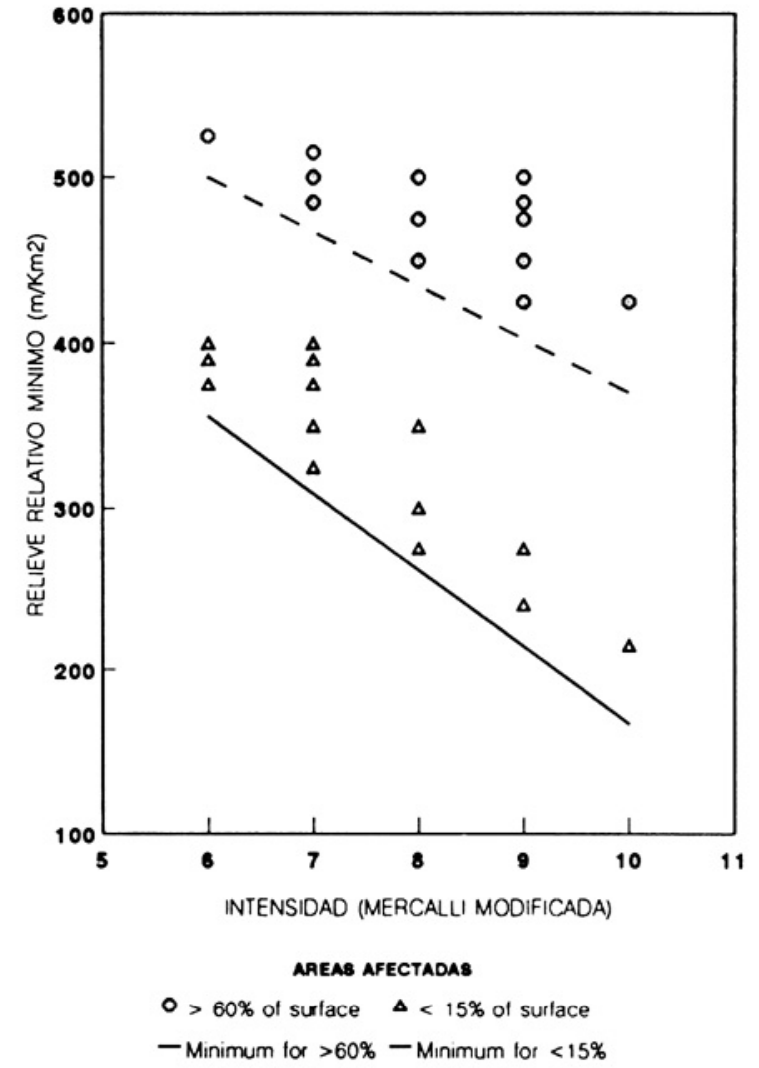

Fig. 5: Areas afectadas por los deslizamientos en relación con los Indices de Relieve Relativo.

Las áreas destruidas por los deslizamientos en relación con la intensidad y magnitud de los sismos

En cada caso el total de las áreas afectadas por los deslizamientos en relación con las tasas de destrucción areal, varían considerablemente según la intensidad y magnitud de los eventos sísmicos. Tal y como se observa en la figura 6 , los sismos que desarrollan intensidades de alrededor de VII pueden ocasionar que en cerca de $90 \mathrm{~km}^{2}$ aparezca al menos un deslizamiento por $\mathrm{km}^{2}$, mientras que en aproximadamente $607 \mathrm{~km}^{2}$ la tasa de destrucción podría ser de $60 \%$. De la misma manera, cuando las intensidades alcanzan o superan IX grados, los deslizamientos aparecen en áreas de al menos $1000 \mathrm{~km}^{2}$ y son capaces de destruir más del $60 \%$ de la superficie de las laderas en alrededor de $90 \mathrm{~km}^{2}$. Por su parte, la figura 7 presenta la relación entre el área afectada y la 
magnitud. Evidentemente, las áreas afectadas aumentan rápidamene con el incremento de la magnitud del sismo.

Ahora bien, cuando la magnitud del sismo alcanza cerca de 5.5 grados es de esperar que hasta unos 3 o $4 \mathrm{~km}^{2}$ sean destruidos en más del $60 \%$, mientras que podría aparecer al menos un deslizamiento por $\mathrm{km}^{2}$ en unos 50 a $60 \mathrm{~km}^{2}$. Si la magnitud alcanza 7,5 grados, los deslizamientos podrian destruir más de un $60 \%$ de la superficie en aproximadamente $70 \mathrm{~km}^{2}$ y ocurrir al menos uno por $\mathrm{km}^{2}$ en más de $1000 \mathrm{~km}^{2}$.

\section{Relaciones entre la distancia epicentral, la magnitud y la destructividad de los deslizamientos}

Es evidente, luego de lo anteriormente discutido, que la aparición y la severidad con que se manifiestan los deslizamientos depende de la

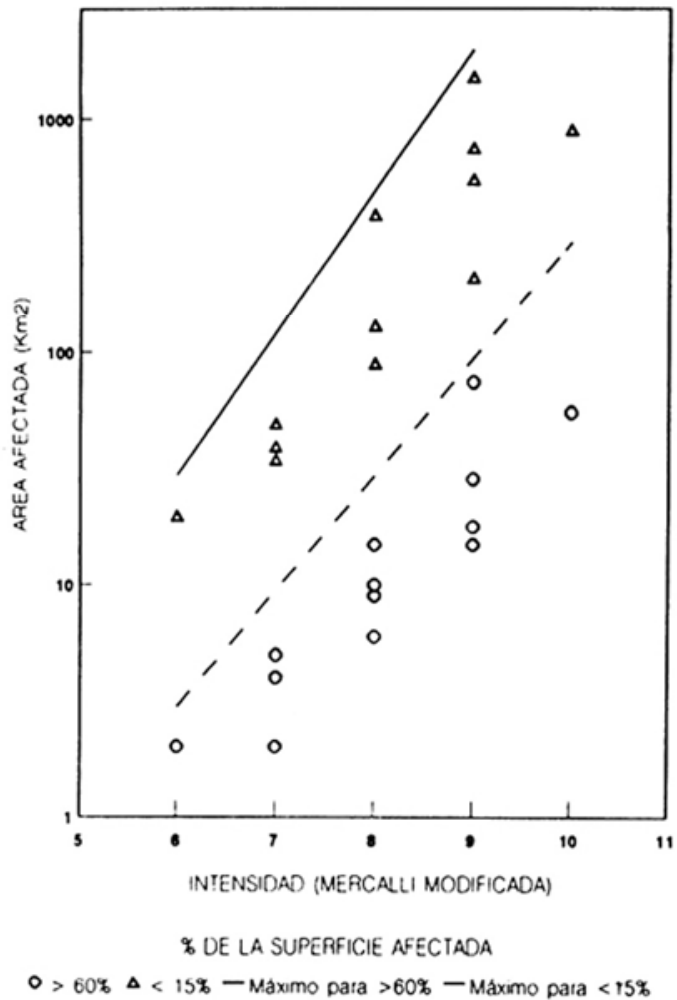

Fig. 6: Relación entre la extensión $\left(\mathrm{en}^{\mathrm{km}} \mathrm{km}^{2}\right.$ de los deslizamientos causados por sismos y las intensidades locales (Mercalli Modificada) en Costa Rica, desde 1888.

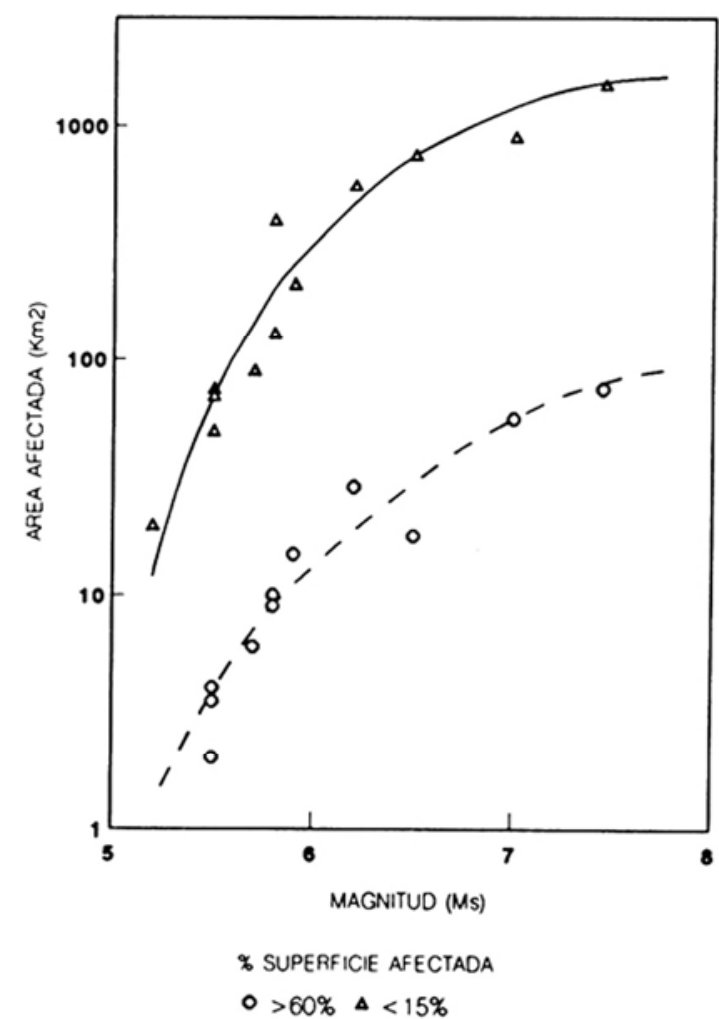

Fig. 7: Extensión de las áreas afectadas por deslizamientos en relación con las magnitudes de los sismos en Costa Rica, desde 1888.

magnitud del sismo y de la distancia epicentral, entre otros factores. Este tema también ha sido discutido por varios autores, según los eventos ocurridos en otras partes del mundo y con los cuales se ha intentado establecer comparaciones.

Por ejemplo, en la figura 8 se muestran varias correlaciones realizadas por diversos autores (Ishihara, 1987; Keefer et al, 1989; Yasuda \& Sugitani, 1988) y en donde también aparecen los datos de Costa Rica (Mora \& Mora, en prensa). Se aprecia una dispersión importante en las observaciones, aunque esto puede deberse a diferencias metodológicas de evaluación y de la manipulación estadistica de la información. En todo caso, se aprecia que la distancia hasta la que aparecen los deslizamientos aumenta rápidamente cuando se comparan magnitudes sismicas entre 5 y 6 grados. El aumento de la distancia tiende hacia una relación semilogarítmicamente linear a partir de una magnitud de 6. 


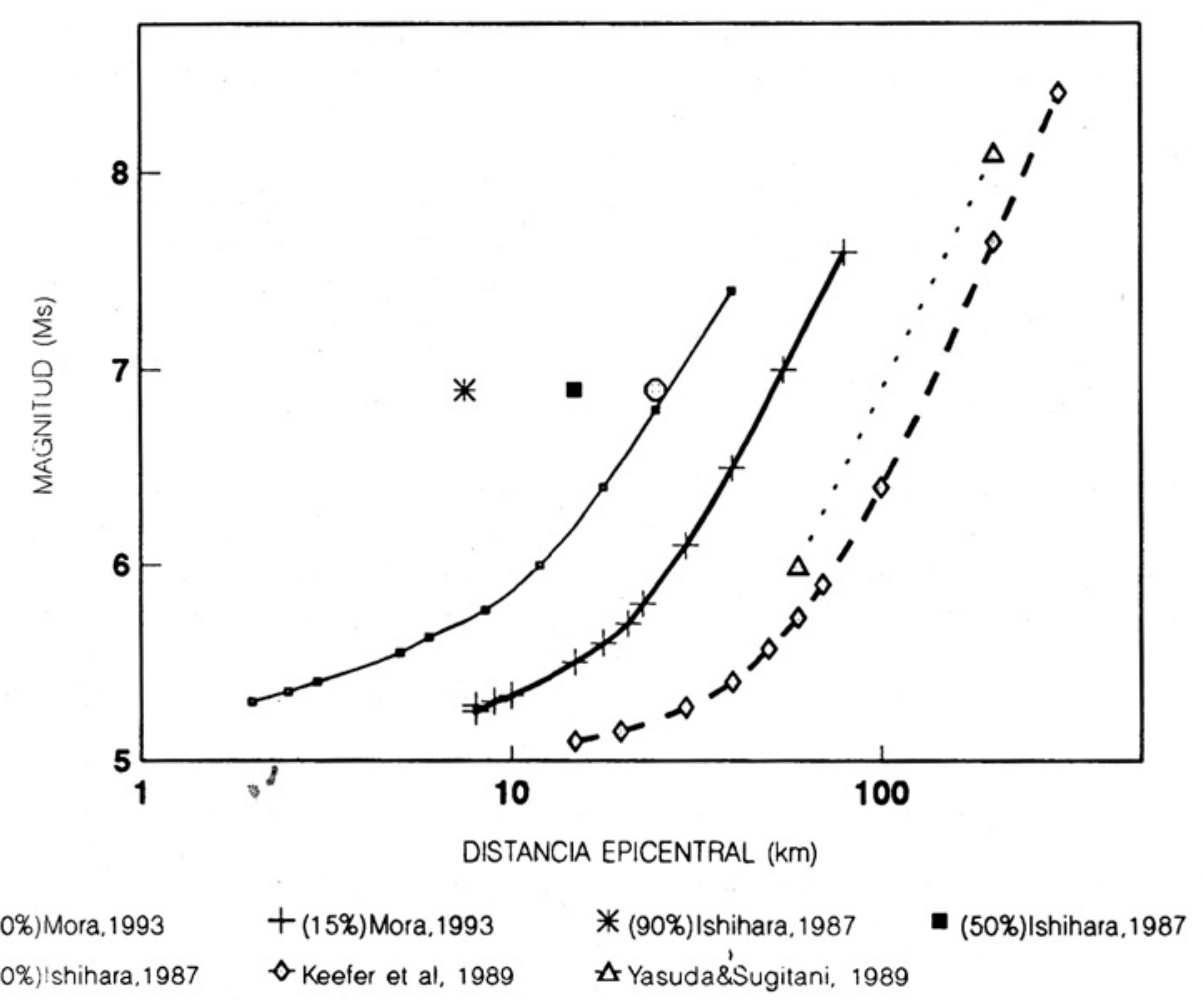

Fig. 8: Relación entre las distancias epicentrales máximas hasta los deslizamientos más distantes y las magnitudes respectivas.

En el caso de Costa Rica, para sismos de magnitud cercana a 5,5, los deslizamientos que son capaces de destruir hasta un $60 \%$ de la superficie pueden aparecer hasta una distancia epicentral de hasta 5 ó $6 \mathrm{~km}$, mientras que los deslizamientos más dispersos aparecen en distancias de hasta unos $20 \mathrm{~km}$. Si la magnitud es más bien de 7.5 grados, los deslizamientos capaces de cubrir más del $60 \%$ del terreno pueden aparecer hasta unos $50 \mathrm{~km}$ de distancia epicentral, mientras que los deslizamientos aislados son capaces de desarrollarse a distancias de poco menos de 100 $\mathrm{km}$.

Estas observaciones han permitido generalizar un modelo que considera las distancias epicentrales máximas a las que se desarrollan los deslizamientos, de acuerdo con la magnitud el sismo y las condiciones de pluviosidad local (Fig. 9). Es interesante resaltar el hecho de que la pluviosidad baja o alta, puede hacer que las distancias a las que se generan los deslizamientos masivos muy destructivos varíe, por ejemplo para una magnitud de 7,0 , entre alrededor de 8 y 25 $\mathrm{km}$, respectivamente. Las distancias máximas también pueden variar entre cerca de $40 \mathrm{~km}$ en regiones secas y $100 \mathrm{~km}$ para las regiones húmedas.

\section{CONCLUSIONES}

Este trabajo ha intentado conocer mejor los factores geodinámicos que controlan la generación de deslizamientos durante los eventos sísmicos. La intención es de aprovechar esta experiencia en la aplicación futura de metodologías de zonificación de la amenaza por deslizamientos de origen sísmico y así facilitar la tarea de los planificadores del uso de la tierra con fines productivos, habitacionales y de conservación.

El Terremoto de Limón, del 22 de abril de 1991, ha sido el más destructivo para Costa Rica, en términos socio-económicos, ambientales y también 
desde el punto de vista de vista de la generación de deslizamientos. Cerca de $2000 \mathrm{~km}^{2}$ fueron afectados por deslizamientos, entre los cuales en cerca de $50 \mathrm{~km}^{2}$ la destrucción de las laderas fue prácticamente completa, en alrededor de $75 \mathrm{~km}^{2}$ la tasa de destrucción alcanzó más de $60 \%$ de la superficie y en casi $1600 \mathrm{~km}^{2}$ se desarrolló al menos un deslizamiento por $\mathrm{km}^{2}$. La mayoría de los deslizamientos ocurridos pueden clasificarse dentro las siguientes categorías:

a) Deslizamientos superficiales del horizonte regolítico ("regolithic flow"): $69 \%$;

b) Desprendimiento y deslizamiento de masas rocosas ("rock-fall slide"): $20 \%$;

c) Deslizamientos traslacionales de masas de suelo y rocas ("rock-earth slumps"): $8 \%$; d) Deslizamientos traslacionales de masas compuestas predominantemente por suelos ("slump-earth flows"): $3 \%$

Los deslizamientos aumentaron considerablemente su actividad e incluso se multiplicaron con las lluvias de alta intensidad de los meses subsiguientes. Esto ocasionó una aceleración considerable de la erosión y la generación de avalanchas de lodo y rocas. Además, se produjeron represamientos en los cauces fluviales de los cuales, al romperse los más grandes, incrementaron los caudales y generaron avenidas que provocaron daños adicionales a la población, líneas vitales y actividades productivas en las regiones bajas.

Las laderas afectadas, en su mayoría, se componen de rocas sedimentarias clásticas

\section{MAGNITUD (Ms)}

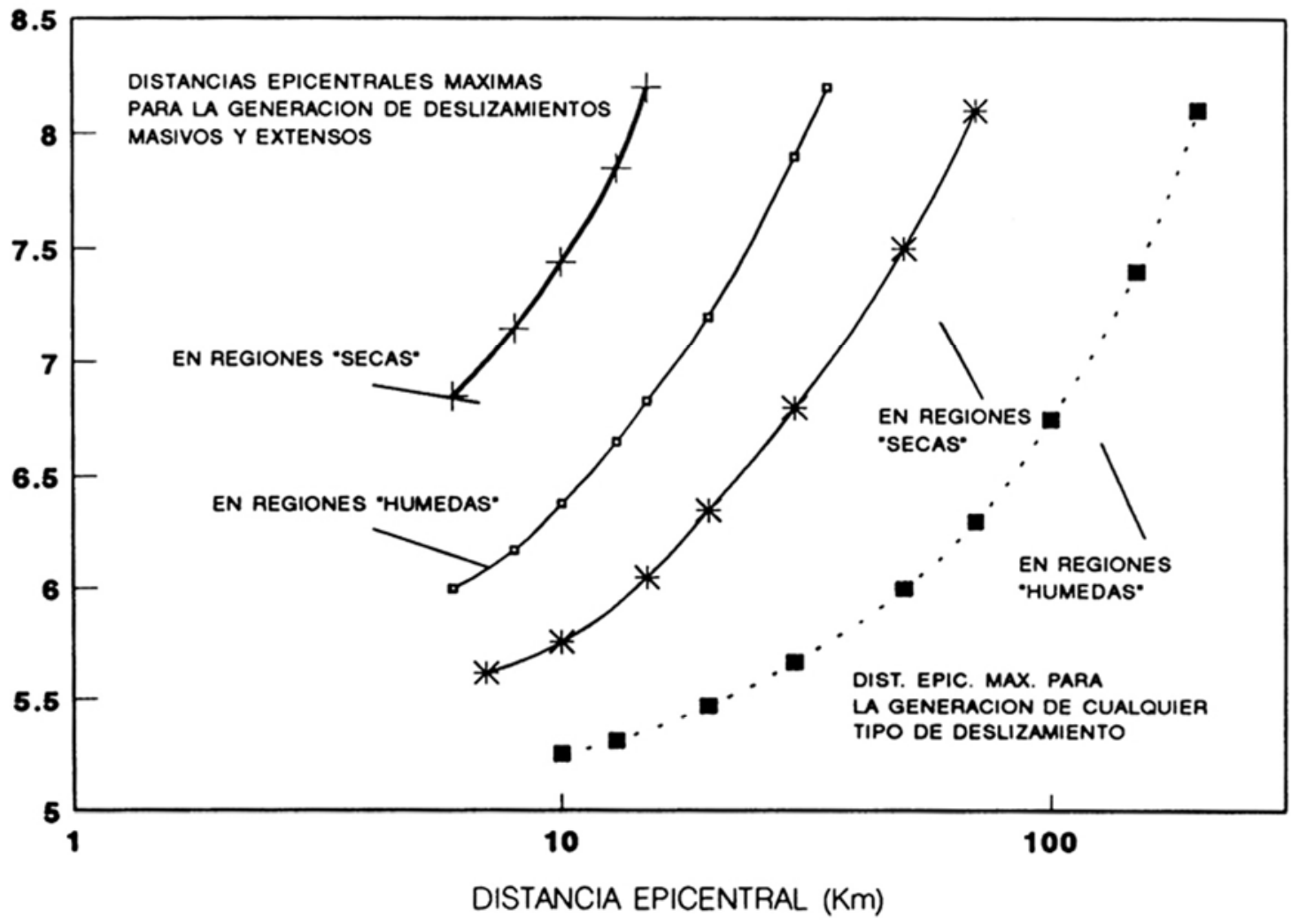

- May.desl.reg.lluvi. + May.desl.reg.seca * Dist máx.reg.seca - Dist.máx.reg.lluvi.

Fig. 9: Relación entre las distancias epicentrales máximas hasta los deslizamientos más distantes y las magnitudes respectivas. 
(areniscas, lutitas) y en menor grado por rocas intrusivas y volcánicas; todas del Terciario y con grados moderados a altos de alteración meteórica e hidrotermal.

Las áreas más afectadas fueron las cuencas altas de los ríos Estrella, Banano y Bananito, además de la cuenca media del río Chirripó. En este último sector, en los alrededores de su confluencia con los ríos Moravia y Boyei (Ayil), se desarrollaron los deslizamientos más espectaculares por sus dimensiones y extensión.

De lo que se ha podido observar y cuantificar, es la condición de la rugosidad del relieve la que parece haber influenciado en mayor grado la generación de deslizamientos. Los relieves superiores de $200 \mathrm{~m} / \mathrm{km}^{2}$ mostraron ser los más propicios para la aparición de deslizamientos. Cuando los relieves alcanzaron valores extremos (hasta $970 \mathrm{~m} / \mathrm{km}^{2}$ ), la extensiø̂n y destructividad de los deslizamientos fue casi total.

$\mathrm{Al}$ comparar este evento sísmico con otros en Costa Rica, en los que igualmente se han generado deslizamientos (desde 1888, 12 eventos), puede observarse que esta tendencia ha sido consistente, aunque influenciada por las variaciones de condición climática y por las características geomorfológicas y litológicas de cada localidad. El tamaño de las áreas totales afectadas, así como las distancias epicentrales hasta las laderas que han generado deslizamientos, dependen de las intensidades, magnitudes y pluviosidad de la localidad, entre otros factores. Lo mismo ha sido constatado en otras regiones del mundo.

\section{REFERENCIAS}

Chacon, T., 1993: Estabilidad de laderas y el impacto del terremoto de Limón. - Tesis de Licenciatura. Escuela de Ciencias Geográficas. Universidad Nacional, Heredia, Costa Rica. 121págs.

Escalante, G., 1983: Mapa geológico preliminar del área sureste de Costa Rica (Cordillera de Talamanca y áreas adyacentes). - Informe inédito.

González, C., 1910: Temblores, terremotos, inundaciones y erupciones volcánicas en Costa Rica. - Imprenta Alsina, San José. 200págs.

Ishihara, K. \& Yasuda, S., 1993: Manual for zonation on seismic geotechnical hazards. - Japanese Soc.Soil
Mechanics and Foundation Engineering. TC4, ISSMFE. 149 págs.

Keefer, D. \& Wilson, R., 1989: Predicting earthquakeinduced landslides with emphasis on arid and semiarid environments. - Publ. Inland Geol. Soc., 2: 118149.

Leandro, G., León, C., Montalto, F., Elizondo, J. \& Chávez, R., 1983: Informe geológico-geotécnico preliminar sobre el sismo de La División, Pérez Zeledón, 3 de julio de 1983. - Comisión Nacional de Emergencia, Departamento de Geología, I.C.E., RECOPE. Informe interno inédito, 69págs.

Mora, S., 1985: Las laderas inestables de Costa Rica. Rev. Geol. América Central, $\underline{3}$ : 131-161.

Mora, S., 1989: Extent and social-economic significance of slope instability in Costa Rica. - En: E. Brabb \& L. Harrod (eds.): Landslides: Extent and economic significance: 93-99. Ed. Balkema, Rotterdam.

Mora, S. \& Morales, L.D., 1986: Los sismos como fuente generadora de deslizamientos en Costa Rica y su impacto sobre las líneas vitales e infraestructura. Memorias I Simposio Latinoamericano de Riesgo Sísmico. C.F.A.I.A., San José, 8págs.

Mora, S. \& Vahrson, W.-G., (en prensa): Macrozonation methodology for landslide hazard determination. Bull. Int. Soc. Eng. Geology.

Mora, S. \& Mora, R., (en prensa): Landslides triggered by the Limón-Telire Costa Rica earthquake and comparaison with other events in Costa Rica. - Prof. Paper, U.S.Geological Survey.

Mora, S., Ishihara, K. Watanabe, H., Yasuda, S. \& Yoshida, N., 1994: Soil liquefaction and landslides during the Limón-Telire Costa Rica Earthquake. Proc. Performance of Ground and Soil Structures During Earthquakes, Special Volume of XIII ICSMFE, New Delhi, India.: 93-101.

Mora, R. \& Salazar, G., 1991: Estado de la cuenca de la quebrada Leona (La Suiza de Turrialba) luego del terremoto del 22 de abril de 1991. - Escuela Centroamericana de Geología, Univ. Costa Rica, Comisión Nacional de Emergencia. Informe interno inédito. 9 págs.

Schuster, R. \& Krizek, R., 1978: Landslides: Análisis and control. - Special Report 176. Transportation Research Board, Commission on Sociotechnical Systems, National Accademy of Sciences. 234 págs.

Vahrson, W.-G. \& Hernandez, G., (en prensa): Geomorphic and hydrologic implications of the 22 April 1993 Earthquake in Limón, Costa Rica. CATENA.

Yasuda, S. \& Sugitani, T., 1988: Case histories of slope failure during past earthquakes in Japan. - Proc. XXIII JSSMFE: .891-892. 